Research Paper

\title{
Role of Amygdala-Infralimbic Cortex Circuitry in Glucocorticoid-induced Facilitation of Auditory Fear Memory Extinction
}

\author{
Abbas Ali Vafaei ${ }^{1}$ (1), Ali Rashidy-Pour ${ }^{1}$ (i), Parnia Trahomi², Samira Omoumi², Masoomeh Dadkhah ${ }^{3 *}$ (i) \\ 1. Research Center of Physiology, Department of Physiology, School of Medicine, Semnan University of Medical Sciences, Semnan, Iran. \\ 2. Student Research Committee, School of Medicine, Semnan University of Medical Sciences, Semnan, Iran \\ 3. Pharmaceutical Sciences Research Center, Ardabil University of Medical Sciences, Ardabil, Iran.
}

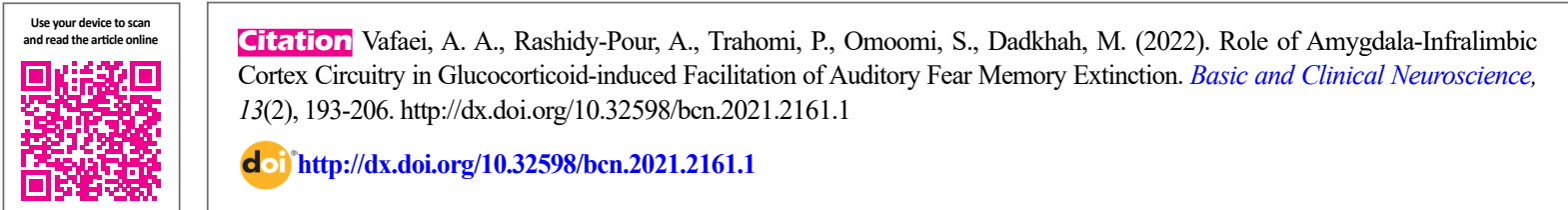

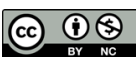

Article info:

Received: 29 Oct 2020

First Revision: 15 Feb 2021

Accepted: 10 Mar 2021

Available Online: 01 Mar 2022

Keywords:

Auditory fear memory, Extinction, Inactivation, Infralimbic, Amygdala

\section{A B S T RA C T}

Introduction: The basolateral amygdala (BLA) and infralimbic area (IL) of the medial prefrontal cortex (mPFC) are two interconnected brain structures that mediate both fear memory expression and extinction. Besides the well-known role of the BLA in the acquisition and expression of fear memory, projections from IL to BLA inhibit fear expression and have a critical role in fear extinction. However, the details of IL-BLA interaction have remained unclear. Here, we investigated the role of functional reciprocal interactions between BLA and IL in mediating fear memory extinction.

Methods: Using lidocaine (LID), male rats underwent unilateral or bilateral inactivation of the BLA and then unilateral intra-IL infusion of corticosterone (CORT) prior to extinction training of the auditory fear conditioning paradigm. Freezing behavior was reported as an index for conditioned fear. Infusions were performed before the extinction training, allowing us to examine the effects on fear expression and further extinction memory. Experiments 1-3 investigated the effects of left or right infusion of CORT into IL and LID unilaterally into BLA on fear memory extinction.

Results: Intra-IL infusion of CORT in the right hemisphere reduced freezing behavior when administrated before the extinction training. Auditory fear memory extinction was impaired by asymmetric inactivation of BLA and CORT infusion in the right IL; however, the same effect was not observed with symmetric inactivation of BLA.

Conclusion: IL-BLA neural circuit may provide additional evidence for the contribution of this circuit to auditory fear extinction. This study demonstrates dissociable roles for right or left BLA in subserving the auditory fear extinction. Our finding also raises the possibility that left BLA-IL circuitry may mediate auditory fear memory extinction via underlying mechanisms. However, further research is required in this area.

\footnotetext{
* Corresponding Author: Masoomeh Dadkhah, PhD.

Address: Pharmaceutical Sciences Research Center, Ardabil University of Medical Sciences, Ardabil, Iran. Tel: +98 (45) 33522437-39

E-mail:m.dadkhah@arums.ac.ir
} 


\section{Highlights}

- Corticosterone infusion in the right (but not the left) infralimbic area facilitates auditory fear memory extinction.

- Corticosterone infusion in the right infralimbic area following symmetric basolateral amygdala inactivation has no effect on auditory fear memory extinction.

- Asymmetric basolateral amygdala inactivation prior to corticosterone infusion into the right infralimbic area impairs auditory fear memory extinction.

\section{Plain Language Summary}

Previous studies have established that glucocorticoids, which are released in stressful conditions, enhance fear memory extinction. In this study, we found that corticosterone infusion into the right infralimbic area, but not the left one, facilitates auditory fear memory extinction. The effect of corticosterone infusion in the infralimbic area was not blocked by the intra-basolateral amygdala injections of lidocaine when administrated in the ipsilateral hemisphere. However, asymmetric basolateral amygdala inactivation and corticosterone infusion into the right infralimbic area impairs auditory fear memory extinction.

\section{Introduction}

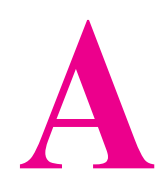

ccumulating evidence indicates that learned fear responses are reduced following repetitive exposure to the conditioned stimulus (CS) without the unconditioned stimulus (US), a process called "fear extinction." This new inhibitory learning has been developed as a translationally valuable assay for studying anxiety and trauma-induced disorders (Sierra-Mercado, Padilla-Coreano, Quirk, 2011; Hitora-Imamura , Miura, Teshirogi, Ikegaya, Matsuki, N., \& Nomura, 2015).

Additionally, the basolateral amygdala (BLA) and medial prefrontal cortex (mPFC), as involved areas in the encoding and expression of learned fear, are part of the neuro-circuitry mechanism underpinning the fear extinction, highlighting the importance of two neural structures in the encoding of learned fear extinction (SotresBayon, Cain, \& LeDoux, 2006; Herry, Ciocchi S, Senn V, Demmou Müller, \& Lüthi. 2008). The evaluation of specific roles of each area revealed that IL and BLA have opposite effects on fear extinction so BLA is thought to be involved in the initial acquisition of extinction (VidalGonzalez, Vidal-Gonzalez, Rauch, \& Quirk 2006; SierraMercado, et al. 2011; Kritman and Maroun 2013).

Previous evidence from functional disconnection studies has investigated the association of memory processing regulation with interactions of BLA with $\mathrm{mPFC}$ (Fuchs, Eaddy, Su, Bell 2007; Mashhoon, Wells, \&
Kantak 2010; Fineberg, et al. 2013). Recent studies have shown that neuronal connections between the BLA and IL regions and reciprocal connections from BLA to IL are essential for fear extinction. On the other hand, plasticity in both the IL and BLA is also involved in fear inhibition (Akirav, Raizel, \& Maroun, 2006; Myers and Davis, 2007; Rahman, Shukla, \& Chattarji, 2018).

Synchronized neural activity in both BLA and mPFC because of the reciprocal connections between two brain areas results in fear memory consolidation (Pape, \& Pare, 2010). Furthermore, by investigating functional disconnection, the regulation of memory processing by interactions between BLA and mPFC has been well documented (Mashhoon, et al. 2010). Any deficits in memory processing after BLA inactivation in one hemisphere and $\mathrm{mPFC}$ in the contralateral hemisphere might be caused by perturbing functional communication within BLAmPFC circuitry. For example, asymmetric inactivation of BLA-mPFC circuitry disrupts serial information flow between these regions and the expression of contextual fear memory (Stevenson, 2011).

A converging body of evidence has established that glucocorticoids can facilitate fear extinction, but GR antagonists impair the extinction of conditioned fear (Ninomiya, Martynhak, Zanoveli, Correia, da Cunha, \& Andreatini 2010; Blundell, Blaiss, Lagace, Eisch, \& Powell 2011; de Quervain, Wolf, \& Roozendaal, 2019). Similarly, extinction-based psychotherapy will be enhanced using cortisol (Aerni, et al. 2004; de Quervain, Schwabe, \& Roozendaal 2017). Furthermore, animal 
studies have shown that facilitating the extinction of contextual fear and fear-potentiated startle can occur following administration of glucocorticoid receptor (GR) agonists (Cai, Blundell, Han, Greene, \& Powell 2006; Yang, Chao, \& Lu, 2006; Ninomiya, et al. 2010; Blundell, et al. 2011).

These effects are mediated partly through the infralimbic (IL) area since intra-IL administration of GR agonists facilitates extinction learning (Dadkhah, Abdullahi, Rashidy-Pour, Sameni, \& Vafaei, 2018). In addition, multiple series of human studies indicate decreased cortisol levels in posttraumatic stress disorder (PTSD) patients (Yehuda 2009), suggesting that low levels of cortisol may contribute to the inability to extinguish fear memory (de Quervain, et al., 2019). Although much of our understanding of fear extinction comes from inactivation studies, the role of functional interactions between BLA and contralateral or ipsilateral IL in mediating the expression of fear memory extinction is unclear. These data support the hypothesis that GRs in both IL and BLA have a role in extinction, but it remains unclear if the activation of GRs in IL depends on BLA.

\section{Materials and Methods}

This present study investigated the role of functional interactions between BLA and IL in mediating the extinction of auditory fear memory. Although the BLA-IL circuitry is well known as a critical circuit in fear memory extinction (Bloodgood, Sugam, Holmes, \& Kash ,2018), reversible inactivation studies should be down to investigate the reciprocal interconnection of BLA-IL circuitry, as well as the modulatory effect of glucocorticoids in these brain structures in regulating the fear memory extinction.

\section{Study animals}

Adult male Wistar rats (weighing 250-280 g) at the time of surgery were used for conducting experiments. The rats were housed (four per cage: $60 \times 40 \times 20 \mathrm{~cm}$ ), maintained in a room with a normal light cycle and temperature $\left(24^{\circ} \mathrm{C} \pm 2^{\circ} \mathrm{C}\right)$, and free access to food and water. All behavioral tests were carried out between 8:00 and 15:00. The Ethics Review Board of Semnan University of Medical Sciences (Iran) approved the experimental protocol.

\section{Surgery and cannulae implantation}

Anesthesia was induced with ketamine hydrochloride $(60 \mathrm{mg} / \mathrm{kg})$ and xylazine $(10 \mathrm{mg} / \mathrm{kg})$ intraperitoneally. At the time of surgery, the rats were placed in a stereotaxic frame, and one 23-gauge stainless steel guide cannula was implanted unilaterally above the IL (anterior,2.9 $\mathrm{mm}$; lateral, $1.0 \mathrm{~mm}$; ventral, $5.0 \mathrm{~mm}$ ) and one above BLA (anterior, $2.8 \mathrm{~mm}$; lateral, $5.0 \mathrm{~mm}$; ventral, - $7.0 \mathrm{~mm}$ below the cortical surface). Dental acrylic was used to fix cannulas to the skull. Stainless steel was inserted into guide cannula and extended $0.5 \mathrm{~mm}$ beyond to prevent clogging. Finally, the rats were given 7 days of postoperative recovery time.

\section{Drug infusions}

Drugs were obtained from Sigma Co., England. Lidocaine (LID) $(10 \mu \mathrm{g} / 0.5 \mu \mathrm{L})$ was infused with microinjection apparatus (Stoelting, USA) into the BLA in one hemisphere to inactivate this region before extinction training. Corticosterone (CORT) $(20 \mathrm{ng} / 0.5 \mu \mathrm{L})$ or vehicle (VEH) were infused into the IL in one hemisphere $10 \mathrm{~min}$ after administration of LID or VEH in the right or left BLA. CORT and LID were dissolved in saline and injected in one hemisphere $(20 \mathrm{ng} / 0.5 \mu \mathrm{L}) 10 \mathrm{~min}$ before the fear extinction training. This concentration has been used in our previous study to examine the effects of CORT on fear extinction (Dadkhah, et al., 2018). The saline solution as a VEH was injected into animals in the control group. After removing the stylus from the guide cannulae, CORT and or VEH were infused $(0.5 \mu \mathrm{L})$ over 1 min via a 30 -gauge injector extending $1.0 \mathrm{~mm}$ from the tip of the guide cannulae. The polyethylene tubing (PE10), connected to a Hamilton microsyringe, was inserted into cannulae for microinjection. The rats received $0.3 \mu \mathrm{L}$ of drug bilaterally in $1 \mathrm{~min}$. After infusion, the cannulae were left in their places for an additional $1 \mathrm{~min}$, preventing reverse flow of the microinfused drug.

\section{Experimental groups}

In experiment 1 , the rats were randomly divided into 4 groups: R/IL/SAL, L/IL/SAL (received SAL in the right or left IL), R/IL/CORT, and L/IL/CORT (received CORT in the right or left IL).

In experiment 2 , the rats were randomly divided into the 4 groups which received infusions unilaterally into IL and BLA: IL/SAL-BLA/SAL (received SAL in the right IL and unilateral BLA), IL/CORT-BLA/SAL (received CORT in the right IL and SAL in unilateral BLA), IL/SAL-BLA/LID (received SAL in right IL and LID in unilateral BLA), and IL/LID-BLA/LID (received LID in right IL and LID in unilateral BLA).

In experiment 3 , the rats were randomly divided into 4 groups that received infusions into IL and BLA contralat- 
erally: IL/SAL-BLA/SAL (received SAL in the right IL and contralateral BLA), IL/CORT-BLA/ SAL (received CORT in the right IL and SAL in contralateral BLA), IL/ CORT-BLA/LID (received SAL in the right IL and LID in contralateral BLA), and IL/LID-BLA/LID (received LID in the right IL and LID in contralateral BLA).

\section{Histology}

After behavioral examination, the rats were killed, and their brains were removed and immersed in fresh $10 \%$ formaldehyde, followed by sectioning into $40-\mu \mathrm{m}$ coronal slices with a microtome. The cannulae locations were examined under a light microscope by hematoxylin and eosin staining (Figure1B).

\section{Behavioral test}

\section{Auditory fear conditioning apparatus}

Auditory fear conditioning and extinction were carried out in the conditioning chamber in a sound-attenuating box. The chamber contained a floor equipped with steel bars that delivered an electric foot shock, which served as the US. A video camera inside the chamber recorded animal behaviors. Freezing behavior was measured as a fear index, which referred to the absence of all movements except for respiration (Fanselow, 1994). At the end of each experimental trial, shock grids and trays under the box were cleaned with a wet towel.

\section{Fear conditioning and extinction}

Briefly, habituation was conducted on the first day (day 0 ) of the behavioral test (the rats underwent 12 tones for $30 \mathrm{~s}$, without shock, 3 min inter-trial interval [ITI] after the first 6 tones) for 9 minutes. On the conditioning day (day 1), each rat housed in the grid box was received three conditioning trials in which a tone (tone: $4 \mathrm{kHz}$; $80 \mathrm{~dB}$ sound pressure level, ITI of $2 \mathrm{~min}$ ) immediately followed by the final tone co-terminated with a mild foot-shock $(0.8 \mathrm{~mA}, 1 \mathrm{~s})$ (Figure 2). The freezing time during the $30 \mathrm{~s}$ tone was reported. The results of extinction training for the 15 tones in the extinction training are represented as 5 blocks (the average of 3 trials).

\section{Statistical analysis}

All obtained data were reported as the Mean \pm SEM and analyzed using a 2-way analysis of variance (ANOVA) with repeated measures and an independent-sample $t$ test followed by Tukey's post hoc comparisons. In all experiments, $\mathrm{P}<0.05$ was considered statistical significance. Also, in the analyses of within-session extinction, the data are expressed as 5 blocks which each block considered as the average of $3 \mathrm{CS}$ presentations per block (extinction blocks).

\section{Results}

Experiment 1: CORT infusion in right IL, but not left IL, facilitating fear memory extinction

\section{Conditioning}

No freezing behavior on the conditioning day was observed during the first CS presentation in the extinction test (prior to US presentation). However, all groups had the same freezing during the second and third CS presentations (Figure 3A), suggesting the groups' uniformity. A 2-way ANOVA with repeated measures (group $\times$ conditioned stimulus) indicated a significant effect of CS (F2, 36=957.968, $\mathrm{P}=0.0001)$, no significant effect of group (F3, 24=10.976, $\mathrm{P}=0.396)$ and of between two factors $(\mathrm{F} 1,24=2.896, \mathrm{P}=0.081)$.

\section{Extinction tests}

The extinction data of all experimental groups are displayed in Figure 3. Analysis with repeated measures (group $\times$ days) indicated a significant effect of group (F1, $18=43.357, \mathrm{P}=0.0001$ ), of days ( 3 testing days: Test 1 , Test 2, and Test 3) (F2, 36=48.248, $\mathrm{P}=0.0001)$ and between two factors $(\mathrm{F} 1,12=2.997, \mathrm{P}=0.005)$. ANOVA with repeated measures analysis for the group comparisons as shown in Figure 3, parts $\mathrm{B}$ and $\mathrm{C}$ indicated a significant difference between SAL/IL and R/IL/CORT groups $(\mathrm{P}=0.0001)$, indicating that unilateral CORT infusion in the right IL decreased freezing levels compared to CORT administration in the left IL.

Experiment 2: Symmetric BLA inactivation and CORT infusion in IL without effect on fear memory extinction

\section{Conditioning}

On the conditioning day, freezing behavior was not observed during the first CS presentation in the extinction box (prior to US presentation). However, all groups showed similar freezing during the second and third CS presentations (Figure 4A), which indicates the groups' uniformity. A 2-way analysis of variance (ANOVA) with repeated measures (group $\times$ conditioned stimulus) indicated a significant effect of CS (F1, 24=238.225, $\mathrm{P}=0.0001)$, no significant effect of group $(\mathrm{F} 3,24=1.897$, $\mathrm{P}=0.157)$ and no significant effect between two factors (F1, 24=05.775, $\mathrm{P}=0.519)$. 


\section{Extinction tests}

According to the extinction data of the experimental groups displayed in Figure 4, and results from 3-way ANOVA with repeated measures (LID $\times$ CORT treatment $\times$ days), a significant effect of LID treatment (F1, 24=65.764, $\mathrm{P}=0.0001)$, of CORT treatment ( $\mathrm{F} 1$, $96=113.097, \mathrm{P}=0.0001$ ), and a significant effect of test days (3 testing days: Test 1, Test 2 and Test 3; F2, $48=64.287, \mathrm{P}=0.0001)$ were observed. This finding further indicates no significant interaction between day and CORT treatments $(\mathrm{F} 1,24=25.992, \mathrm{P}=0.0001)$, no significant interaction between the LID and days (F1, 24=7.313, $\mathrm{P}=0.012$ ), of treatment and LID (F1, 24=65.764, $\mathrm{P}=0.0001$ ), and significant interaction between CORT treatment and LID and days $(\mathrm{F} 1,24=7.313, \mathrm{P}=0.012)$.

Post hoc comparisons indicate a decrease in freezing behavior in all groups after $15 \mathrm{CS}$ presentations on Test 1, Test 2, and Test 3 (Figure 3B and 3C). The SAL/ILLID/BLA, CORT/IL-SAL/BLA groups continued to display decreased freezing during the three test sessions compared to the SAL/IL-SAL/BLA group $(\mathrm{P}=0.0001)$, indicating that unilateral CORT infusion in IL or unilateral BLA inactivation in the same hemisphere facilitates extinction. Furthermore, there was no significant difference between the CORT/IL-SAL/BLA and CORT/ IL-LID/BLA groups on the blocks of three tests. This finding indicates that unilateral CORT infusion in IL has a facilitatory effect on fear extinction, and this effect is independent of BLA activation in the same hemisphere (Figure 4B and 4C).

Experiment 3: Asymmetric BLA inactivation and CORT infusion in IL impairing fear memory extinction

\section{Conditioning}

Like the previous experiments, no freezing behavior was observed during the first CS presentation in the extinction box (prior to US presentation). However, similar freezing during the second and third CS presentations was observed in the groups (Figure 5A), which indicates the groups' uniformity. A 2-way analysis of ANOVA with repeated measures (group $\times$ conditioned stimulus) indicated a significant effect of CS (F1, 24=788.667, $\mathrm{P}=0.0001)$, no significant effect of group ( $\mathrm{F} 3,24=4.133$, $\mathrm{P}=0.017$ ), and no significant effect between two factors (F3, 24=3.275, $\mathrm{P}=0.089$ ).

\section{Extinction tests}

Three-way ANOVA with repeated measures $(\mathrm{LID} \times \mathrm{CORT}$ treatment $\times$ days $)$ indicated a significant effect of LID (F1, 24=18.458, $\mathrm{P}=0.0001)$, a significant effect of CORT treatment (F1, 96=33.829, $\mathrm{P}=0.0001)$, and a significant effect of days ( 3 testing days: Test 1 , Test 2 and Test 3) (F2, 48=101.428, $\mathrm{P}=0.0001)$, which further indicates no significant interaction between day and CORT treatments (F2, 48=2.772, $\mathrm{P}=0.109$ ), no significant interaction between the LID and days (F2, $48=4.189, \mathrm{P}=0.052$ ), no significant interaction between CORT treatment and LID (F1, 24=96.504, P=0.0001), and significant interaction between CORT treatment, LID, and days (F1, 24=0.572, $\mathrm{P}=0.457)$.

Decreased freezing behavior was observed in all groups after 15 CS presentations on Test 1, Test 2, and Test3 (Figure 5B and 5C). The SAL/IL-LID/BLA, CORT/ IL-SAL/BLA groups continued to display decreased freezing during the test sessions compared to the SAL/ IL-SAL/BLA group $(\mathrm{P}=0.0001)$, indicating that both unilateral CORT infusion in IL and unilateral BLA inactivation, have a facilitatory effect on extinction. Furthermore, a significant difference was observed between the CORT/IL-SAL/BLA and CORT/IL-LID/BLA groups on the blocks of Test 1 , Test 2 , and Test $3(\mathrm{P}=0.003)$. This finding indicates that while unilateral CORT infusion in IL (right IL) and contralateral BLA inactivation both facilitate fear extinction, CORT infusion in IL following ipsilateral inactivation of BLA impairs fear extinction (Figure 5B and 5C). In other words, CORT infusion in IL facilitates fear extinction via BLA activation in the contralateral hemisphere (Figure 5B and 5C).

\section{Discussion}

In the present study, we investigated the role of functional interactions between BLA and IL in mediating auditory fear memory extinction. Our results of experiment 1 suggest that unilateral CORT infusion into the IL subregion facilitates the extinction of auditory fear memory when administrated in the right IL. In experiment 2 , we showed that symmetric BLA inactivation prior to CORT infusion in the right IL was ineffective on auditory fear memory extinction. In experiment 3 , we observed that asymmetric BLA inactivation and then CORT infusion in right IL impaired auditory fear memory extinction suggesting that right and left BLA play different roles in mediating the facilitatory effect of GRs of IL on fear memory extinction. 
CORT infusion in right IL facilitating auditory fear memory extinction

Glucocorticoids are released from the adrenal cortex and have an essential role in regulating different kinds of learning and memory, including auditory and contextual fear memory and different brain regions such as the hippocampus, basolateral amygdala (BLA), and mPFC (Abrari, Rashidy-Pour, Semnanian, \& Fathollahi 2009; Barsegyan, Mackenzie, Kurose, McGaugh, \& Roozendaal, 2010) mediates underlying mechanisms. Additionally, it is essential to note that glucocorticoids play a key role in fear memory processing (Okuda, Roozendaal, \& McGaugh, 2004) and facilitate the consolidation of extinction fear learning (Cai, et al. 2006; Abrari, Rashidy-Pour, Semnanian, \& Fathollahi, 2008).

The present study indicates that whereas infusion of CORT in left IL does not affect fear extinction, the intrainfusion of CORT in the right IL reduces fear expression, similar to the pattern from our previous study, indicating that IL plays an important role in CORT-induced facilitation of fear memory extinction (Dadkhah, et al. 2018). The extinction of shock-induced fear memories depends on corticosterone synthesis (Abrari, et al., 2008). However, these effects are mediated via the IL sub-region since glucocorticoid receptor (GR) agonist infusion into IL results in the facilitation of extinction learning while blocking of a GR antagonist suppresses extinction learning (Dadkhah, et al. 2018). Consistent with this finding, a recent study implicated that IL is contributed to decreased fear responses during extinction (SierraMercado, et al., 2011). Thus, IL is a key modulator for the extinction of fear memory and suggests that this area might be critical for treating anxiety disorders through pharmacological manipulations.

CORT infusion in right IL following symmetric BLA inactivation, not disturbing fear memory extinction

Another remarkable finding of the current study lies in clarifying the role of functional interactions between BLA and IL in mediating the extinction of auditory fear memory. We found that symmetric inactivation of BLA and CORT infusion in the right IL leaves fear memory extinction intact. In line with these findings, previous studies demonstrate that the impairment of fear memory expression following reversible inactivation of BLA agrees with other studies (Laurent \& Westbrook 2009).

Moreover, the effects of inactivating BLA and CORT infusion in the IL area were examined separately. The logic of the underlying strategy was raised from the reciprocal connections between BLA and IL that display increased firing associated with fear extinction or facilitation of memory extinction, respectively (Senn, et al. 2014). Our findings support and extend the previous study that used functional disconnection to express unilateral or ipsilateral connectivity between these structures and assumed that unilateral inactivation has no effect functionally in each area (Stevenson, 2011).

To our knowledge, this research was the first to directly examine whether projections from IL to BLA are predominantly ipsilateral or contralateral and facilitatory effect of CORT on memory extinction is mediated via ipsilateral or contralateral BLA. A considerable body of work using techniques, including inactivation or ILtargeted pharmacological manipulation, has shown that IL activity is critical to successful fear extinction, but functionally disrupting this region impacts fear memory impairment (Likhtik \& Paz, 2015; Tovote, Fadok, \& Lüthi 2015). Furthermore, interactions between BLA and $\mathrm{mPFC}$ and reciprocal connections between BLA and IL might subserve fear memory extinction (Bloodgood, et al., 2018). Reciprocal projections between BLA and IL lead to plasticity in IL, suggesting the involvement of BLA and IL in mediating the extinction memory (SierraMercado, et al., 2011). Most importantly, synchronized neural activity in the BLA-mPFC circuit after fear learning also indicates a differential requirement of the ILBLA circuit in memory processing (Stevenson, 2011). Given the well-established role of IL projections in the extinction (Hikind \& Maroun 2008), it has been shown that fear extinction requires IL cortex projections to the BLA (Bloodgood, et al., 2018).

The glutamatergic projections from the mPFC interact with amygdala gamma-aminobutyric acid (GABA) ergic neurons (Paré, Quirk, \& Ledoux, 2004), and these synapses may provide inhibitory input to the amygdala So these projections to intercalated cells could be a possible pathway by which IL tone responses can suppress the expression of conditioned fear (e.g., reduce freezing) (Rosenkranz \& Grace, 2002).

While IL sends descending projections to BLA, and BLA receives reciprocal connections, it is unclear whether projections from IL to BLA are predominantly unilateral or bilateral. In addition, the strongest pathways from the BLA to the $\mathrm{mPFC}$ originated from different neurons (Reppucci \& Petrovich, 2016). Concerning the functional connection between these regions and especially the BLA-IL circuit (Popa, Duvarci, S., Popescu, Léna, Paré, 2010; Contreras \& Gutiérrez-García 2019), the results 

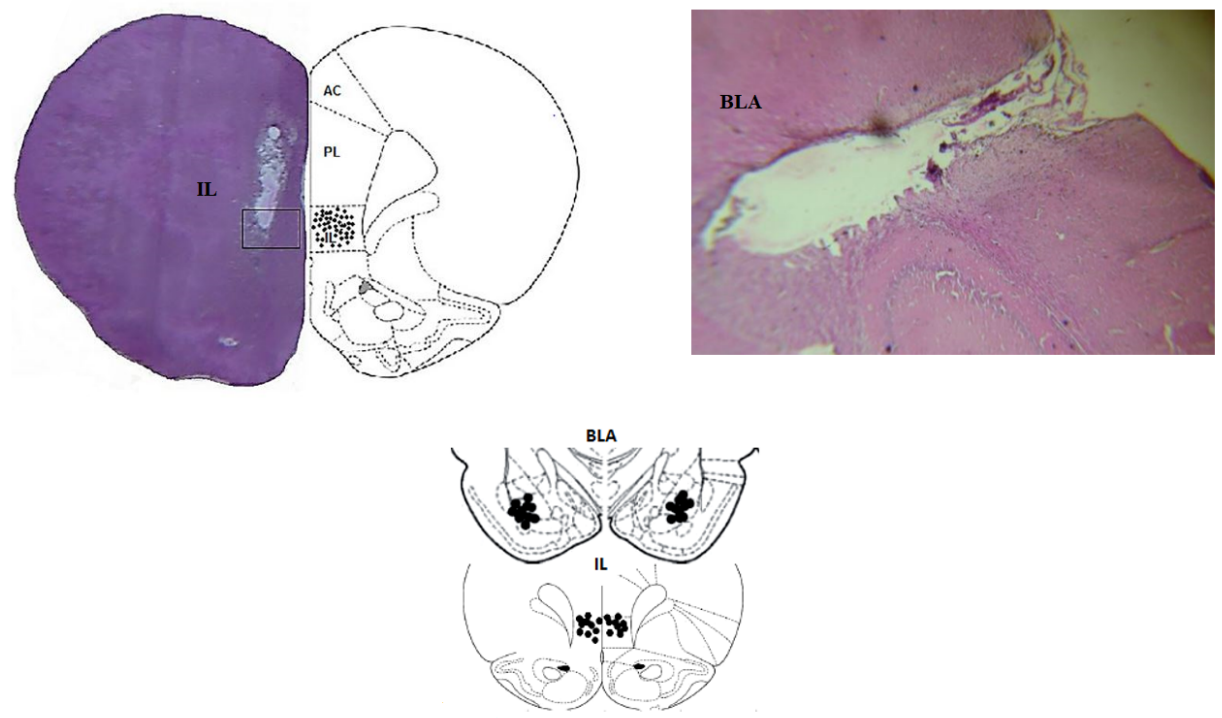

Figure 1. Experimental Timeline of Behavioral Training, Testing Design, and Drug Injections

NEUR SCIENCE

show that unilateral BLA inactivation alone has no effect on the facilitatory effect of intra-IL CORT infusion on fear extinction. One possibility is that the effect of CORT infusion into IL on extinction memory expression would act via BLA in different hemispheres. So, this finding suggests that the processing in the IL-BLA circuit in fear memory extinction is not parallel. Here, functional disconnection also supposes that unilateral inactivation of BLA alone has no effect on facilitatory effect of intra-IL CORT infusion on fear extinction, supporting a lateralized function of amygdala and mPFC fear memory processing (Stevenson, 2011). Overall, functional interactions between BLA and IL might be an essential issue for subserving extinction fear memory expression.

As mentioned above, BLA and mPFC share ipsilateral connections. Consistent with the findings of this prior study, we found that intra-IL CORT infusion following unilateral BLA inactivation in the same hemisphere leaves fear extinction intact compared to the control group, which received intra-IL CORT alone. This finding indicates that asymmetric BLA inactivation impure that BLA and mPFC share ipsilateral connections between two areas. The current study found no effects of symmetric BLA inactivation and intra-IL CORT infusion on the extinction behavior in the auditory fear condition test, confirming the serial connection in previous findings.

Asymmetric BLA inactivation and CORT infusion in IL impairing fear memory extinction

The finding of impaired auditory fear memory extinction with the intra-IL infusion of CORT in the right hemisphere following asymmetric BLA-inactivation provided the subserved functional interactions in the BLA-IL circuit in processing the auditory fear memory extinction. This finding suggests the different reciprocal nature of connections of BLA with IL in CORT-induced extinction memory facilitation via CORT administration in IL in brain hemispheres.

This finding suggests that amygdala stimulation induced by IL activation during fear extinction is associ-

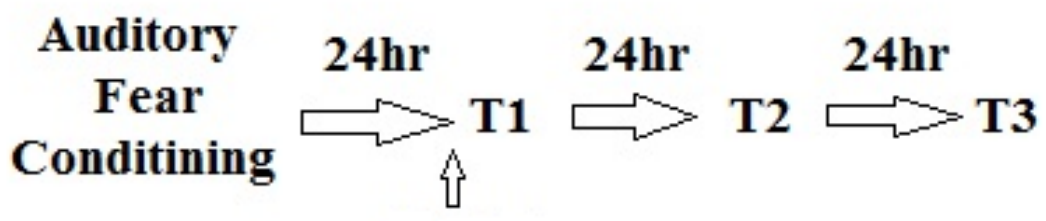

\section{IL \& BLA Infusion}

Figure 2. A Histological Verification of Guide Cannulae Placements in the Experimental Subjects

A) IL: Infralimbic, B). BLA: Basolateral Amygdala (See Materials and Methods for more detail) 


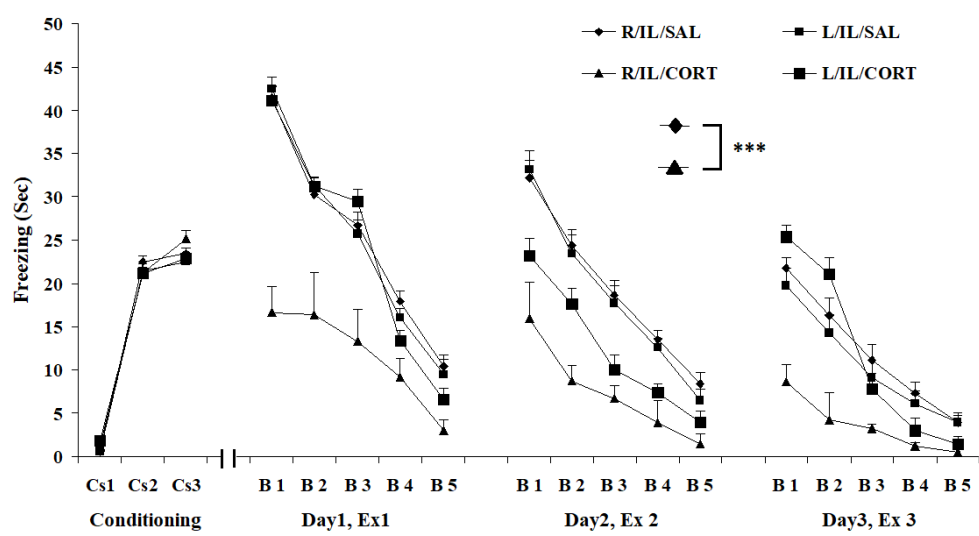

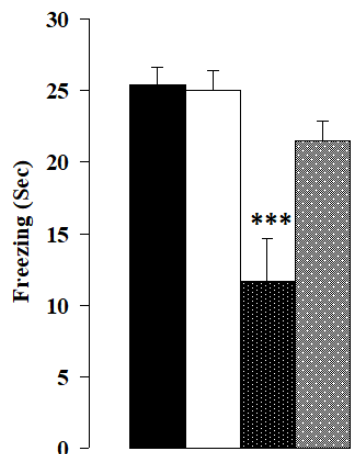

Day1, Ex1

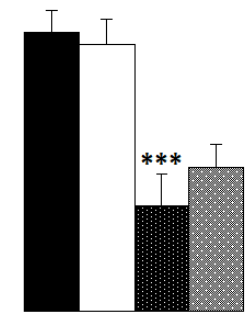

T2

Day2, Ex2

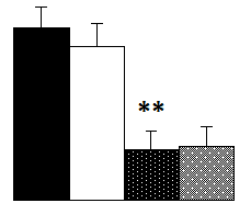

T3

Day3, Ex3

NEUR SCIENCE

Figure 3. Effects of Intra-IL (Right or Left IL) Infusion of Corticosterone on Fear Memory Extinction, A) No Differences in Freezing Response Between the Groups During the Conditioning, B, C) Freezing During Fear Extinction Test

Unilateral infusion of CORT into right IL significantly decreased freezing compared to the saline (SAL) group $\left({ }^{* * *} \mathrm{P}<0.001\right)$.

Abbreviations: R, right; B, block; IL, infralimbic; BLA, basolateral amygdala; LID, lidocaine; CORT, corticosterone; SAL, saline; Ex, extinction.

ated with the facilitatory effect of CORT infusion in IL, and this effect depends on the inhibition of amygdala neurons which increases freezing levels. As noted before, GABAergic mechanisms in the amygdala play a critical role in controlling the extinction of fear.

In addition, vmPFC is widely considered to inhibit the amygdala through activating the group of GABAergic intercalated cells ITC in BLA, which also have extinction neurons (Seo, et al. 2016). However, the effects of inactivation of the BLA, which impaired extinction memory, agrees with other studies (Demetrio Sierra-Mercado). Moreover, glucocorticoid effects on memory processing are associated with the bidirectional interactions between the BLA and mPFC. The memory-modulating properties of glucocorticoids depend on functional interactions between the mPFC and BLA (Roozendaal, McReynolds, Van der Zee, Lee, McGaugh, \& McIntyre, 2009).
Furthermore, neurotransmitters have a critical role in fear extinction because an increase in dopamine (DA) and noradrenaline (NA) levels in the mPFC (Hugues, Garcia, \& Léna 2007) has been observed, and increasingly appears, the increase in DA in the $\mathrm{MPFC}$ results in a greater reduction in fear after extinction training. Despite this fact, evidence reported changing flux of neurotransmitters, including DA, NA, serotonin (5-HT), GABA, and glutamate in key brain regions during the extinction of fear memories (Cahill \& Milton 2019). Thus, it could be argued that impaired fear extinction following asymmetric BLA inactivation and CORT infusion in IL, at least in part, is due to changing flux of neurotransmitters in the IL-BLA circuit.

Another reason for the functional disconnection between BLA and IL might be associated with the inactivation of rostral or caudal BLA (Floresco \& Ghods-Sharifi 2007; Fuchs, et al. 2007). In support of this possibility, a 


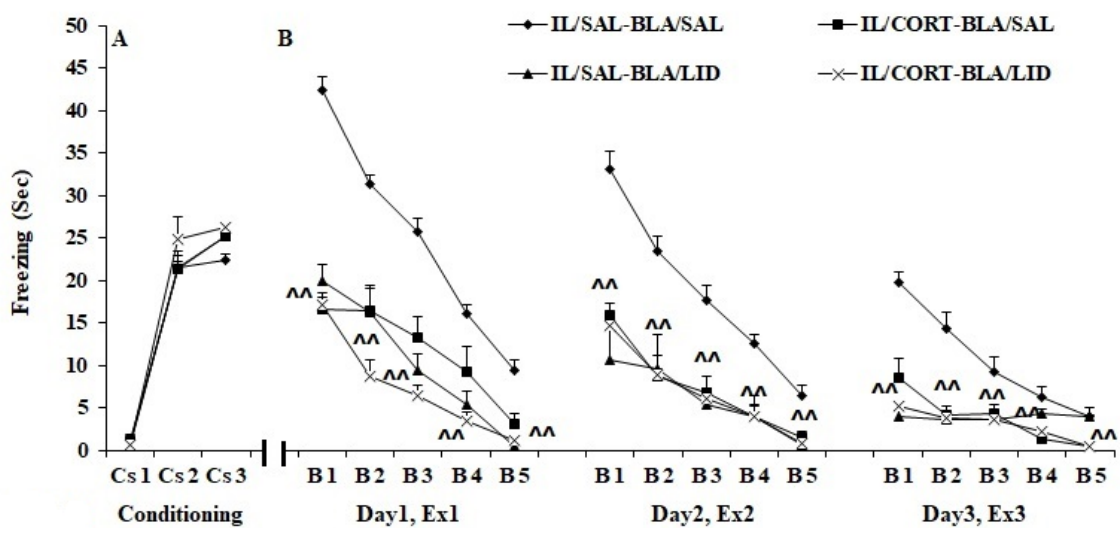

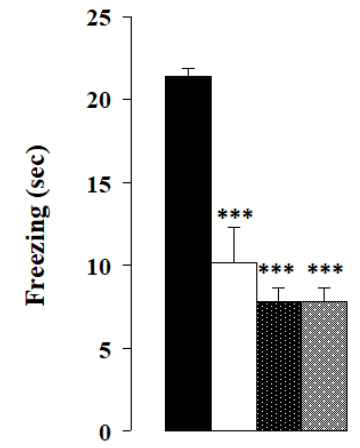

T1

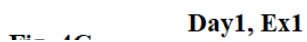

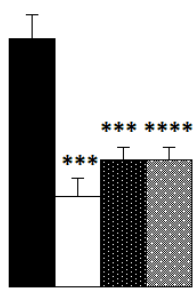

T2

Day2, Ex2

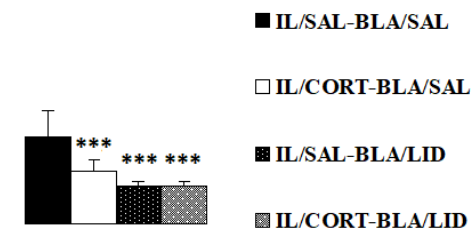

T3

Day3, Ex3

NEUR SCIENCE

Figure 4. Effects of Symmetric BLA Inactivation and Then Corticosterone Infusion Into Right IL on Fear Memory Extinction, A) No Observed Significant Difference in Freezing Between the Groups During the Conditioning, B, C) Freezing During Fear Extinction Testing

Compared to IL/SAL-BLA/LID group, symmetric BLA inactivation and then corticosterone infusion into the right IL had the same effect on freezing levels. ${ }^{* *} \mathrm{P}<0.001$ compare to IL/SAL-BLA/SAL group.

Abbreviations: R, right; B, block; IL, infralimbic; BLA, basolateral amygdala; LID, lidocaine; CORT, corticosterone; SAL, saline; Ex, extinction.

similar effect was also reported after functionally disconnecting of posterior BLA and paralimbic sub-region of $\mathrm{mPFC}$ results which play a critical role in fear memory expression, resulting from impairment by asymmetric inactivation of pBLA and PL (Stevenson, 2011).

It should be noted that earlier findings have shown that brain lateralization may affect memory function (Baker $\&$ Kim, 2004). We found that either the intra-IL infusion of CORT in the right hemisphere and contralateral or ipsilateral BLA inactivation did not have similar effects, again demonstrating the existence of lateralization. Different effects of unilateral BLA inactivation in each hemisphere on freezing behavior in the present study indicate that the asymmetric neural activity of the left BLA in auditory fear extinction which depends on the intra-IL infusion of CORT in the right hemisphere.
Consistent with the findings of this experiment, earlier studies provide further support for the notion that inactivation of left or right BLA does not have similar effects due to lateralization. Furthermore, it has been shown that right BLA is dominant in fear memory conditioning (Baker \& Kim, 2004). Reports from previous studies demonstrate that interactions between BLA and mPFC are involved in regulating memory processing by using functional disconnection (Fuchs, Eaddy, et al. 2007; Mashhoon, Wells, et al. 2010). In this procedure, any resulting deficits caused by memory processing following BLA inactivation in one hemisphere and $\mathrm{mPFC}$ in the contralateral one might be related to perturbed communication within BLA-mPFC circuitry. One logical reason for the underlying strategy is that although unilateral inactivation, which leaves each area functionally unaffected, impairments in the serial information flow in 


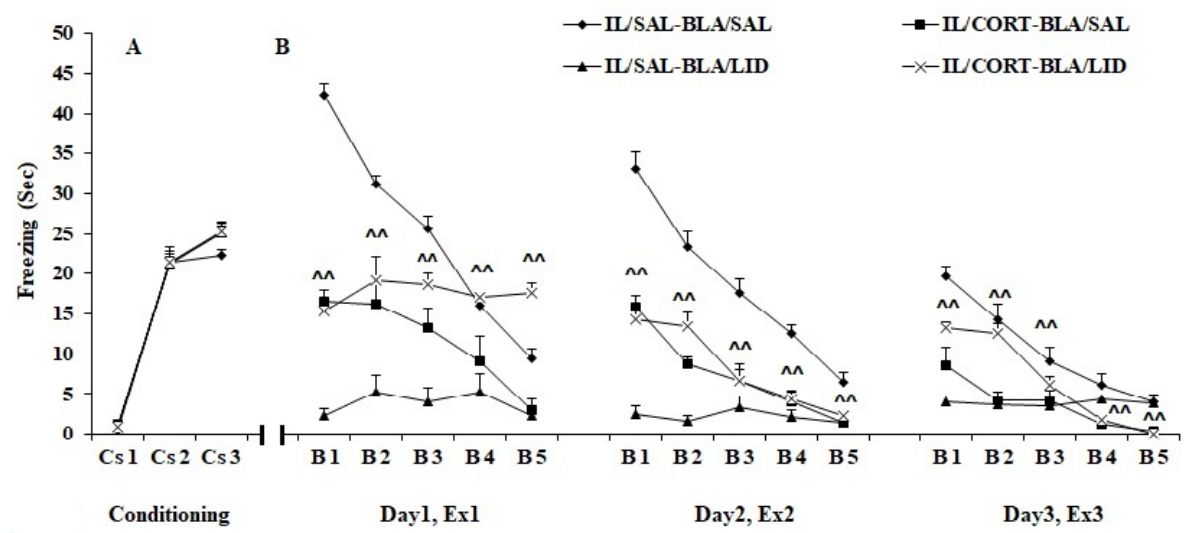

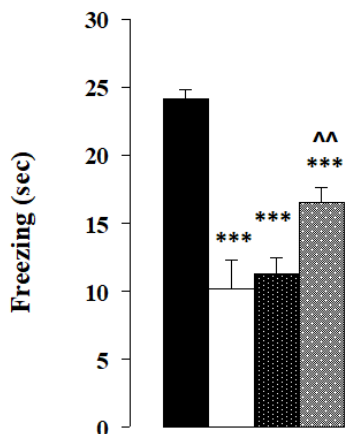

T1

Day1, Ex1

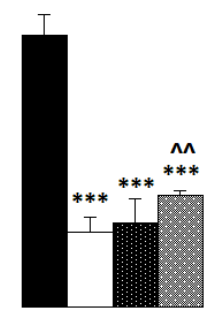

T2

Day2, Ex2

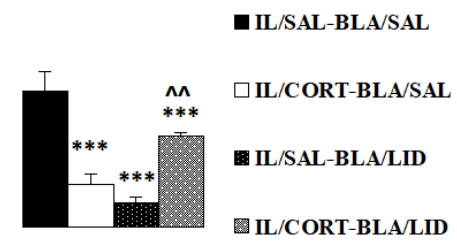

T3

Day3, Ex3

Figure 5. Effects of Asymmetric BLA Inactivation and Then Corticosterone Infusion Into Right IL on Fear Memory Extinction, A) No Differences in Freezing Levels Between the Groups During the Conditioning, B, C) Freezing During Fear Extinction Testing

Compared to IL/SAL-BLA/LID group, asymmetric BLA inactivation and then unilateral infusion of intra-right IL CORT increased freezing. ${ }^{* *} \mathrm{P}=0.0001$ compared to IL/SAL-BLA/SAL group and $\wedge \wedge \wedge \mathrm{P}=0.0001$ compared to IL/SAL-BLA/LID group

Abbreviations: R, right; B, block; IL, infralimbic; BLA, basolateral amygdala; LID, lidocaine; CORT, corticosterone; SAL, saline; Ex, extinction.

BLA-mPFC circuitry are observed by asymmetric inactivation (Stevenson, 2011).

Many more inputs originated from different areas project to the amygdala (Pape \& Pare 2010). Thus, the activity of IL-projecting cells in the BLA, which is related to extinction, might emerge due to reciprocal connectivity from the IL back down onto these cells.

With respect to hemisphere-specific morphological changes in BLA, dendritic retraction has been shown in the left BLA in response to extinction (Moench, Maroun, Kavushansky, \& Wellman 2016). Further, increased connectivity between BLA-ventromedial prefrontal cortex (vmPFC) during extinction learning has been reported (Belleau, Pedersen, Miskovich, Helmstetter, \& Larson 2018). Previous studies have documented direct connections between the vmPFC and the BLA, supporting a bottom-up control mechanism due to direct connections between the centromedial nucleus of the amygdala (CeMA) and vmPFC (Seo, et al., 2016).

These findings propose a fundamental role for the left BLA in CORT-induced facilitation of fear memory extinction when administrated in the right IL.

\section{Conclusion}

Functional connections between the IL and BLA have received less attention in the literature. The present study demonstrated the presence of contralateral IL and BLA connections, which have not been previously documented. We also confirmed that the well-known lateralization connections assume a noticeable difference in hemisphere-specific processing between the left and right amygdalae. 


\section{Ethical Considerations}

\section{Compliance with ethical guidelines}

All protocols were approved by the Ethics Committee of the Semnan University of Medical Sciences.

\section{Funding}

This research was supported by grant from Semnan University of Medical Sciences.

\section{Authors' contributions}

Conceptualization, investigation, writing - original draft, and data collection: Masoomeh Dadkhah; Data analysis: Masoomeh Dadkhah; Writing - review \& editing: Abbas Ali Vafaei, Ali Rashidy-Pour, data collection: Parnia Tarahomi, Samira Omoumi.

\section{Conflict of interest}

The authors declared no conflicts of interest.

\section{Acknowledgments}

We thank the staff of Laboratory of Learning and Memory, Research Center of Physiology, Department of Physiology, School of Medicine, Semnan University of Medical Sciences, Semnan, Iran.

\section{References}

Abrari, K., Rashidy-Pour, A., Semnanian, S., \& Fathollahi, Y (2008). Administration of corticosterone after memory reactivation disrupts subsequent retrieval of a contextual conditioned fear memory: Dependence upon training intensity. Neurobiology of Learning and Memory, 89(2), 178-184. [DOI:10.1016/j.nlm.2007.07.005] [PMID]

Abrari, K., Rashidy-Pour, A., Semnanian, S., \& Fathollahi, Y. (2009). Post-training administration of corticosterone enhances consolidation of contextual fear memory and hippocampal long-term potentiation in rats. Neurobiology of Learning and Memory, 91(3), 260-265. [DOI:10.1016/j.nlm.2008.10.008]

Aerni, A., Traber, R., Hock, C., Roozendaal, B., Schelling, G., \& Papassotiropoulos, A., et al. (2004). Low-dose cortisol for symptoms of posttraumatic stress disorder. American Journal of Psychiatry, 161(8), 1488-1490. [DOI:10.1176/appi. ajp.161.8.1488] [PMID]

Akirav, I., Raizel, H., \& Maroun, M. (2006). Enhancement of conditioned fear extinction by infusion of the GABAA agonist muscimol into the rat prefrontal cortex and amygdala. European Journal of Neuroscience, 23(3), 758-764. [DOI:10.1111/j.14609568.2006.04603.x] [PMID]
Baker, K. B., \& Kim, J, J. (2004). Amygdalar lateralization in fear conditioning: Evidence for greater involvement of the right amygdala. Behavioral Neuroscience, 118(1), 15-23. [DOI:10.1037/0735-7044.118.1.15]

Barsegyan, A., Mackenzie, S. M., Kurose, B, D., McGaugh, J, L., \& Roozendaal, B. (2010). Glucocorticoids in the prefrontal cortex enhance memory consolidation and impair working memory by a common neural mechanism. Proceedings of the National Academy of Sciences, 107(38), 16655-16660. [DOI:10.1073/ pnas.1011975107]

Belleau, E. L., Pedersen, W. S., Miskovich, T. A., Helmstetter, F J., \& Larson, C. L. (2018). Cortico-limbic connectivity changes following fear extinction and relationships with trait anxiety. Social Cognitive and Affective Neuroscience, 13(10), 1037-1046. [DOI:10.1093/scan/nsy073] [PMID] [PMCID]

Bloodgood, D. W., Sugam, J. A., Holmes, A., \& Kash, T. L. (2018). Fear extinction requires infralimbic cortex projections to the basolateral amygdala. Translational Psychiatry, 8(1), 60. https://www.nature.com/articles/s41398-018-0106-x

Blundell, J., Blaiss, C. A., Lagace, D. C., Eisch, A. M., \& Powell, C, M. (2011). Block of glucocorticoid synthesis during re-activation inhibits extinction of an established fear memory. Neurobiology of Learning and Memory, 95(4), 453-460. [DOI:10.1016/j. nlm.2011.02.006] [PMID] [PMCID]

Cahill, E. N., \& Milton, A. L. (2019). “Neurochemical and molecular mechanisms underlying the retrieval-extinction effect." Psychopharmacology, 236(1): 111-132. [DOI:10.1007/s00213018-5121-3] [PMID] [PMCID]

Cai, W. H., Blundell, J., Han, J., Greene, R. W, \& Powell, C. M (2006). Postreactivation glucocorticoids impair recall of established fear memory. Journal of Neuroscience, 26(37), 9560-9566. [DOI:10.1523/JNEUROSCI.2397-06.2006] [PMID] [PMCID]

Contreras, C. M., \& Gutiérrez-García, A. G. (2019). Reciprocal interactions between the basolateral amygdala and infralimbic and prelimbic regions of the mPFC: Actions of diazepam. Neuroscience Letters, 704, 78-83. [DOI:10.1016/j.neulet.2019.03.051] [PMID]

Dadkhah, M., Abdullahi, PR., Rashidy-Pour, A., Sameni, H R., \& Vafaei, A. A. (2018). Infralimbic dopamine D2 receptors mediate glucocorticoid-induced facilitation of auditory fear memory extinction in rats. Brain Research, 1682, 84-92. [DOI:10.1016/j.brainres.2018.01.006] [PMID]

de Quervain, D., Schwabe, L., \& Roozendaal, B. (2017). Stress, glucocorticoids and memory: Implications for treating fearrelated disorders. Nature Reviews. Neuroscience, 18(1), 7-19. [DOI:10.1038/nrn.2016.155] [PMID]

de Quervain, D., Wolf, O. T., \& Roozendaal, B. (2019). Glucocorticoid-induced enhancement of extinction-from animal models to clinical trials. Psychopharmacology, 236(1),183-199. [DOI:10.1007/s00213-018-5116-0] [PMID] [PMCID]

Fanselow, M. S. (1994). Neural organization of the defensive behavior system responsible for fear. Psychonomic Bulletin $\mathcal{E}$ Review, 1(4), 429-438. [DOI:10.3758/BF03210947] [PMID]

Fineberg, N. A., Haddad, P. M., Carpenter, L., Gannon, B., Sharpe, R., \& Young, A. H., et al. (2013). The size, burden and cost of disorders of the brain in the UK. Journal of Psychopharmacology, 27(9), 761-770. [DOI:10.1177/0269881113495118] [PMID] [PMCID] 
Floresco, S. B. \& Ghods-Sharifi, S. (2007). Amygdala-prefrontal cortical circuitry regulates effort-based decision making Cerebral Cortex, 17(2), 251-260. [DOI:10.1093/cercor/bhj143] [PMID]

Fuchs, R. A., Eaddy, J. L., Su, Z. I., \& Bell, G. H. (2007). Interactions of the basolateral amygdala with the dorsal hippocampus and dorsomedial prefrontal cortex regulate drug context-induced reinstatement of cocaine-seeking in rats. The European Journal of Neuroscience, 26(2), 487-498. [DOI:10.1111/ j.1460-9568.2007.05674.x] [PMID]

Herry, C., Ciocchi S, Senn V, Demmou L, Müller C, \& Lüthi A. (2008). Switching on and off fear by distinct neuronal circuits. Nature, 454(7204), 600-606. [DOI:10.1038/nature07166] [PMID]

Hikind, N. \& Maroun, M. (2008). Microinfusion of the D1 receptor antagonist, SCH23390 into the IL but not the BLA impairs consolidation of extinction of auditory fear conditioning. Neurobiology of Learning and Memory, 90(1), 217-222. [DOI:10.1016/j.nlm.2008.03.003] [PMID]

Hitora-Imamura, N., Miura, Y., Teshirogi, C., Ikegaya, Y., Matsuki, N., \& Nomura, H. (2015). Prefrontal dopamine regulates fear reinstatement through the downregulation of extinction circuits. Elife, 4, e08274. [DOI:10.7554/eLife.08274]

Hugues, S., Garcia, R., \& Léna, I. (2007). Time course of extracellular catecholamine and glutamate levels in the rat medial prefrontal cortex during and after extinction of conditioned fear. Synapse, 61(11), 933-937. [DOI:10.1002/syn.20448] [PMID]

Kritman, M., \& Maroun, M. (2013). Inhibition of the PI3 kinase cascade in corticolimbic circuit: Temporal and differential effects on contextual fear and extinction. International Journal of Neuropsychopharmacology, 16(4), 825-833. [DOI:10.1017/ S1461145712000636] [PMID]

Laurent, V. \& Westbrook, R. F. (2009). Inactivation of the infralimbic but not the prelimbic cortex impairs consolidation and retrieval of fear extinction. Learning $\mathcal{E}$ Memory, 16(9), 520-529. [DOI:10.1101/lm.1474609] [PMID]

Likhtik, E., \& Paz, R. (2015). Amygdala-prefrontal interactions in (mal) adaptive learning. Trends in Neurosciences, 38(3), 158-166. [DOI:10.1016/j.tins.2014.12.007] [PMID] [PMCID]

Mashhoon, Y., Wells, A. M., \& Kantak, K. M. (2010). Interaction of the rostral basolateral amygdala and prelimbic prefrontal cortex in regulating reinstatement of cocaine-seeking behavior. Pharmacology Biochemistry and Behavior, 96(3), 347-353. [DOI:10.1016/j.pbb.2010.06.005] [PMID] [PMCID]

Moench, K. M., Maroun, M., Kavushansky, A., \& Wellman, C. (2016). Alterations in neuronal morphology in infralimbic cortex predict resistance to fear extinction following acute stress. Neurobiology of Stress, 3, 23-33. [DOI:10.1016/j.ynstr.2015.12.002] [PMID] [PMCID]

Myers, K. M. \& Davis, M. (2007). Mechanisms of fear extinction. Molecular Psychiatry, 12(2), 120-150. [DOI:10.1038/ sj.mp.4001939]

Ninomiya, E. M., Martynhak, B. J., Zanoveli, J. M., Correia, D., da Cunha, C., \& Andreatini, R. (2010). Spironolactone and low-dose dexamethasone enhance extinction of contextual fear conditioning. Progress in Neuro-Psychopharmacology and Biological Psychiatry, 34(7), 1229-1235. [DOI:10.1016/j.pnpbp.2010.06.025] [PMID]
Okuda, S., Roozendaal, B., \& McGaugh, J. L. (2004). Glucocorticoid effects on object recognition memory require training-associated emotional arousal. Proceedings of the National Academy of Sciences, 101(3), 853-858. [DOI:10.1073/pnas.0307803100] [PMID] [PMCID]

Pape, H. C., \& Pare, D. (2010). Plastic synaptic networks of the amygdala for the acquisition, expression, and extinction of conditioned fear. Physiological Reviews, 90(2), 419-463. [DOI:10.1152/physrev.00037.2009] [PMID] [PMCID]

Paré, D., Quirk, G. J., \& Ledoux, J. E. (2004). New vistas on amygdala networks in conditioned fear. Journal of Neurophysiology, 92(1), 1-9. [DOI:10.1152/jn.00153.2004] [PMID]

Popa, D., Duvarci, S., Popescu, A. T., Léna, C., Paré, D. (2010). Coherent amygdalocortical theta promotes fear memory consolidation during paradoxical sleep. Proceedings of the $\mathrm{Na}$ tional Academy of Sciences, 107(14), 6516-6519. [DOI:10.1073/ pnas.0913016107] [PMID] [PMCID]

Rahman, M. M., Shukla, A., \& Chattarji, S. (2018). Extinction recall of fear memories formed before stress is not affected despite higher theta activity in the amygdala. Elife, 7, e35450. [DOI:10.7554/eLife.35450] [PMID] [PMCID]

Reppucci, C. J. \& Petrovich, G. D. (2016). Organization of connections between the amygdala, medial prefrontal cortex, and lateral hypothalamus: A single and double retrograde tracing study in rats. Brain Structure and Function, 221(6), 2937-2962. [DOI:10.1007/s00429-015-1081-0] [PMID] [PMCID]

Roozendaal, B., McReynolds, J. R., Van der Zee, E. A., Lee, S., McGaugh, J. L., \& McIntyre, C. K. (2009). Glucocorticoid effects on memory consolidation depend on functional interactions between the medial prefrontal cortex and basolateral amygdala. Journal of Neuroscience, 29(45), 14299-14308. [DOI:10.1523/JNEUROSCI.3626-09.2009] [PMID] [PMCID]

Rosenkranz, J. A. \& Grace, A. A. (2002). Cellular mechanisms of infralimbic and prelimbic prefrontal cortical inhibition and dopaminergic modulation of basolateral amygdala neurons in vivo. Journal of Neuroscience, 22(1), 324-337. [DOI:10.1523/ JNEUROSCI.22-01-00324.2002] [PMID] [PMCID]

Senn, V., Wolff, S. B. E., Herry, C., Grenier, F., Ehrlich, I., \& Gründemann, J., et al. (2014). Long-range connectivity defines behavioral specificity of amygdala neurons. Neuron, 81(2), 428-437. [DOI:10.1016/j.neuron.2013.11.006] [PMID]

Seo, D, O., Funderburk, S. C., Bhatti, D. L., Motard, L. E., Newbold, D., Girven, K.S., et al. (2016). A GABAergic Projection from the Centromedial Nuclei of the Amygdala to Ventromedial Prefrontal Cortex Modulates Reward Behavior. The Journal of Neuroscience, 36(42), 10831-10842. [DOI:10.1523/ JNEUROSCI.1164-16.2016] [PMID] [PMCID]

Sierra-Mercado, D., Padilla-Coreano, N., Quirk, G. J. (2011). Dissociable roles of prelimbic and infralimbic cortices, ventral hippocampus, and basolateral amygdala in the expression and extinction of conditioned fear. Neuropsychopharmacology, 36(2), 529-538. [DOI:10.1038/npp.2010.184] [PMID] [PMCID]

Sotres-Bayon, F., Cain, C. K., \& LeDoux, J. E. (2006). Brain mechanisms of fear extinction: Historical perspectives on the contribution of prefrontal cortex. Biological Psychiatry, 60(4), 329336. [DOI:10.1016/j.biopsych.2005.10.012] [PMID]

Stevenson, C. W. (2011). Role of amygdala-prefrontal cortex circuitry in regulating the expression of contextual fear 
memory. Neurobiology of Learning and Memory, 96(2), 315-323. [DOI:10.1016/j.nlm.2011.06.005] [PMID]

Tovote, P., Fadok, J. P., \& Lüthi, A. (2015). Neuronal circuits for fear and anxiety. Nature Reviews Neuroscience, 16(6), 317. [DOI:10.1038/nrn3945] [PMID]

Vidal-Gonzalez, I., Vidal-Gonzalez, B., Rauch, S. L., \& Quirk, G. J. (2006). Microstimulation reveals opposing influences of prelimbic and infralimbic cortex on the expression of conditioned fear. Learning \& Memory, 13(6), 728-733. [DOI:10.1101/ lm.306106] [PMID] [PMCID]

Yang, Y. L., Chao, P.K., \& Lu, K. T. (2006). Systemic and intraamygdala administration of glucocorticoid agonist and antagonist modulate extinction of conditioned fear. Neuropsychopharmacology 31(5), 912-924. [DOI:10.1038/sj.npp.1300899] [PMID]

Yehuda, R. (2009). Status of glucocorticoid alterations in posttraumaticstress disorder. Annals of the New York Academy of Sciences, 1179(1), 56-69. [DOI:10.1111/j.1749-6632.2009.04979.x] [PMID] 
This Page Intentionally Left Blank 